\title{
A Better, Faster Road From Biological Data to Human Health: A Systems Biology Approach for Engineered Cell Cultures
}

\author{
Brian T. Hawkins and Sonia Grego
}

Traditionally, the interactions of drugs and toxicants with human tissue have been investigated in a reductionist way-for example, by focusing on specific molecular targets and using single-cell-type cultures before testing compounds in whole organisms. More recently, systems biology approaches attempt to enhance the predictive value of in vitro biological data by adopting a comprehensive description of biological systems and using sophisticated computational tools that can deal with the complexity of these systems. However, the utility of computational models resulting from these efforts completely relies on the quality of the data used to construct them.

Here, we propose that recent advances in the development of bioengineered, 3D, multicellular constructs provide in vitro data of sufficient complexity and physiological relevance to be used in predictive systems biology models of human responses. Such predictive models are essential to maximally leveraging these emerging bioengineering technologies to improve both therapeutic development and toxicity risk assessment.

This brief outlines the opportunities presented by emerging technologies and approaches for the acceleration of drug development and toxicity testing, as well as the challenges lying ahead for the field.

\section{What Is Systems Biology?}

- The data-based, computational, and mathematical modeling of complex biological systems and their dynamic behavior

- A paradigm in antithesis to reductionist approaches of biological response focused on "one gene, one receptor, one mechanism"

- Driven by the advent of high-throughput bioassays and increased computational power that enable bioinformatics analysis of -omics data

- Network-centric view, with focus on mathematical description of signaling, transcriptional, and metabolic networks and their interactions

- Aims at discovering network-level properties not evident from the study of individual components (emergent properties)

\section{Key Issues and Opportunities}

- Because of the limitations of animal models and in vitro assays, the pipeline for new therapeutics grows smaller and chemical toxicity screening is failing to meet the growing demand for hazard assessment.

- Engineered cellular constructs provide access to physiologically relevant in vitro data of sufficient quality for improved predictive modeling of human responses.

- Sophisticated computational tools are needed to translate in vitro biological data to actionable information about health effects of bioactive compounds. 


\section{New Technologies Are Needed to Drive Therapeutic Development and Toxicant Screening in the 21st Century}

The cost of drug development continues to increase ( $>1.2$ billion per drug), even as the pipeline for new therapeutics grows increasingly smaller. It has been proposed that " $t]$ he decline in the productivity of the pharmacological industry may stem partly from the underestimation of the complexity of cells, organisms and human disease."' With the goal of developing more effective ways to treat disease and prolong health, approaches that leverage new technologies have emerged. For example, precision medicine aims at health care that considers individual variability in genes, environment, and lifestyle. A parallel set of challenges exists in toxicology, where the introduction of novel chemicals into industrial, agricultural, and household use has outpaced the ability of regulatory entities to thoroughly assess their potential hazards. In response, efforts have been made to employ high-throughput screening and computational modeling for more efficient classification and prioritization of chemical risks. ${ }^{2}$ However, these efforts to date have focused largely on compound screening by cell monocultures and molecular targets.

\section{Advances in Bioengineering Have the Potential to Revolutionize In Vitro Biology}

Engineered cell cultures are biomimetic "organotypic" cellular constructs that represent key functional units of living human organs. Key features include a 3D microarchitecture defined by the spatial distribution and arrangement of cells in their "native" environments, tissue-tissue interfaces, tissue functionalities such as barrier properties and muscle contraction, and organ-specific interactions of cells with their physical microenvironments. These constructs, by better reflecting the complexity of native tissue, may recapitulate its emergent properties. For example, recent studies in constructs containing multiple cell types have demonstrated that heterotypic cell-cell interactions underlie responses that are not observed in monocultures. ${ }^{3-5}$

This field, which combines cell biology and microfabrication technologies, has grown quickly thanks to the support of largescale federal programs. Multiple engineered organ models (e.g., liver, lung, heart, brain, kidney, bone, muscle) have been demonstrated. To date, development of these constructs has been limited primarily to their characterization, and only a few studies have demonstrated the amenability of engineered constructs for pharmacological ${ }^{6}$ or toxicological ${ }^{4}$ testing applications. In vitro cardiac myocyte cultures for cardiac adverse effects screening are being evaluated by the US Food and Drug Administration (FDA) for regulatory approval according to an updated testing paradigm, the Comprehensive In Vitro Proarrhythmia Assay (CiPA). ${ }^{7}$ Other platforms have become commercially available, such as the HepatoPac ${ }^{\oplus}$ system for liver toxicity screening, which demonstrated good predictivity for drug-induced liver injury in a 45-drug test set, particularly when human cells were used. ${ }^{8}$ Despite these early promising outcomes, the recapitulation of tissue functionalities by many constructs remains largely untested, and their predictive performance against current models remains to be validated. ${ }^{9}$

A long-term goal is to incorporate organotypic cell cultures into precision medicine applications by using induced pluripotent stem cells to generate patient- and populationspecific organs-on-chips. ${ }^{10}$ However, in vitro data do not directly emulate clinical observations, and several challenges remain in translating insight from in vitro systems to in vivo outcomes.

\section{Systems Biology Manages Complexity Across Scales}

Biological systems are inherently complex and nonlinear given the multiplicity of molecular pathways, their interactions across spatial and temporal scales, and their regulation by parallel, redundant, and feedback loop processes. Addressing this "multiscale problem" is the core of systems biology, as reflected in efforts to define mathematical relationships between perturbations (drug/toxicant exposure, genotypic variation, environmental interactions) at the molecular/cellular levels and physiological consequences of those perturbations (drug action, toxic response, disease) that manifest at tissue, organ, and organism levels. In pharmacology and toxicology, which focus on dose- and time-dependent responses, the systems biology approach seeks to extend the description of differential gene expression to a quantification of network-level perturbations. ${ }^{11}$

Because it is not feasible to take all components of a complex system into account, systems biology uses various approaches to reduce complexity, most notably networkbased mathematical descriptions, ${ }^{11}$ and takes advantage of both deterministic and probabilistic methods ${ }^{12}$ to account for the influence of incomplete knowledge. For example, differential equations are used in pharmacokinetic/ pharmacodynamic (PK/PD) modeling of absorption, distribution, and metabolism. This deterministic approach has been used successfully for in silico models for in vitro to in vivo extrapolation in pharmaceutical development, as evinced by low drug attrition due to PK-related issues. For modeling and predicting more complex cellular behaviors such as proliferation (cancer) and cytokine production (inflammation), the dynamics of multiple signal pathways must 
be integrated, and a high degree of uncertainty is introduced due to limited knowledge of the regulatory biochemistry and limited experimental data at the needed scales. For such phenomena, modeling using probabilistic, stochastic methods is more predictive than purely deterministic models. ${ }^{13}$

The systems biology approach can offer advantages in cases in which purely experimental approaches would take too many resources. This utility is perhaps best demonstrated in combinatorial drug studies. For example, Morris and colleagues recently showed good predictivity of target kinase activation in hepatocellular carcinoma cells stimulated by various combinations of cytokines and in the presence of many different drug combinations, using a "constrained fuzzy logic" modeling approach. ${ }^{14}$ Importantly, they also demonstrated that deviations of the biological data from the model predictions could be used to identify previously unknown relationships among signaling pathways, thus guiding further experiments and subsequent model refinement. ${ }^{14}$ Another example of the utility of a systems biology approach is the use of a feedback system control platform, which has been exploited for the rapid optimization of dozens of drug combinations out of billions of possibilities, aimed at new therapeutic regimens in a macrophage infection model of tuberculosis. ${ }^{15}$

\section{Bioengineering Integrated with Systems Biology- Based Modeling Is a Mutually Beneficial Relationship}

We posit that before bioengineered cell culture technology can find precision medicine applications, a predictive, accurate model of human responses must be developed combining physical models of tissue and an across-scale systems biology approach.

Access to quality data at the right biological scales is key. The development of sophisticated multicellular models offers access to in vitro response data in physiologically relevant settings and at a higher hierarchical level than ever before, as illustrated in Figure 1. Efforts are ongoing to demonstrate the feasibility of physically integrating multiple organs into one system-“human on a chip" - to capture even higher-level data in vitro. The ability to create a computational model capturing data across scales from molecular-level pathways to tissue-level (and possibly higher) responses will provide unprecedented comprehensiveness in the prediction of human responses. ${ }^{16}$

The development of computational tools and bioengineered constructs is not typically integrated. An iterative approach is needed, with computational modeling both informing the design of experiments to be conducted with bioengineered constructs and extrapolating key in vitro data to in vivo outcomes. The ideal computational model will show us how to get better data rather than simply more data. Early steps toward this goal have been undertaken in a study of network modeling of global gene expression profiles in tendon and cartilage cells in vitro to compare comprehensively the differentiation of these cells in monolayer cultures and 3D cultures to the native tissue; this approach can be used to guide design and refinement of engineered cultures in a rigorous and evidence-based manner. ${ }^{17}$ Conversely, the biologically rich, high-quality in vitro data from organotypic constructs will serve as the indispensable backbone for the development of a computational testbed that will enable more accurate and predictive modeling of the human response to a drug or toxicant. ${ }^{16}$

Human physiology is far too complex to be modeled fully using current computing technology. More powerful computing capacity is needed to create models large enough to incorporate pathways of response and to operate across biological scales. Appropriate software platforms for such models may need to include specialized architectures geared toward life sciences applications and to incorporate hybrid systems that merge deterministic models of higher-level processes with the probabilistic, network-based approaches used to model biological pathways.

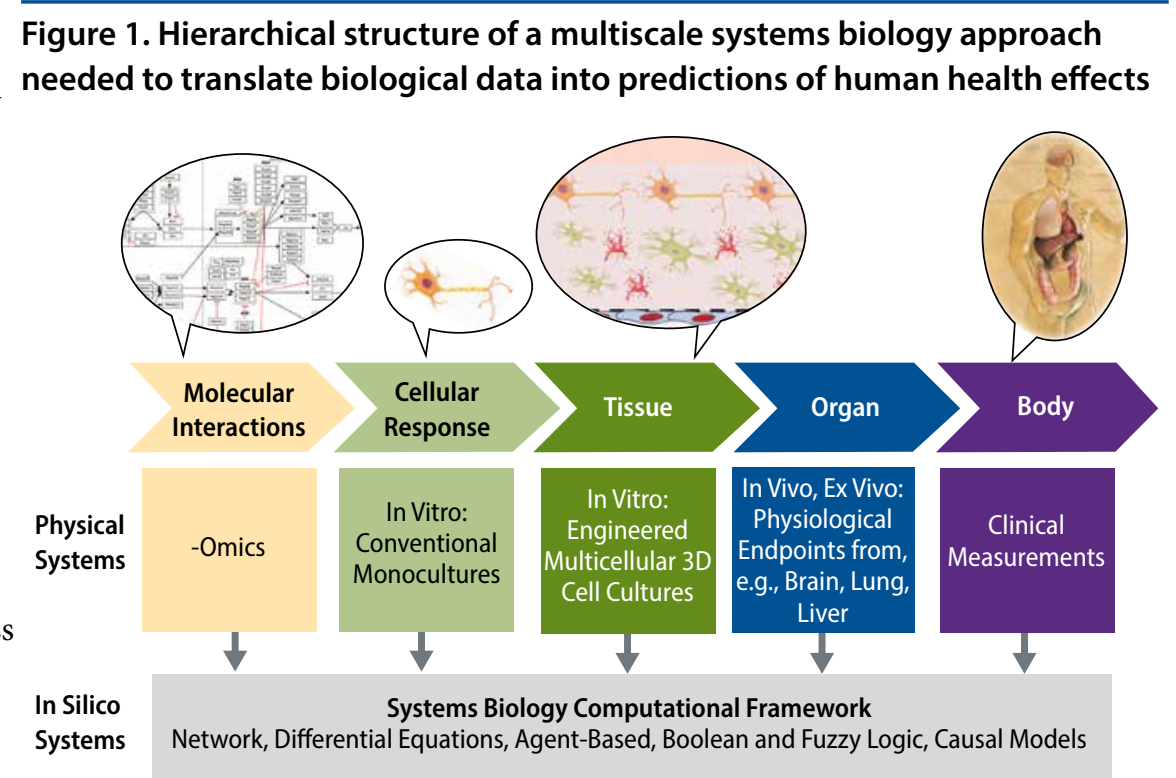

Source: The pathway image in the Molecular Interactions bubble is used under the Creative Commons CCO Waiver and has been reprinted from http://www.wikipathways.org/index.php/Pathway:WP358 


\section{Future Directions}

The integration of bioengineering with systems biology-based modeling has tremendous potential to accelerate toxicity screening and therapeutic development. Some of the greatest added value is anticipated in addressing diseases and toxicant responses that develop over time and involve multiple biological pathways and biological scales. The integrated approach can better address chronic and degenerative diseases with complex clinical presentations and high care costs. For example, better models of inflammatory responses in specific organs may enable treatments that address the underlying causes of conditions including asthma, chronic obstructive pulmonary disease, and neurodegenerative diseases-including the potential roles of environmental toxicants in the onset of disease-rather than simply treating their symptoms. Similarly, incorporation of patient-specific cells into physiologically relevant constructs would enable more powerful predictive modeling of individual responses to cancer drugs and their combinations to optimize therapeutic intervention (personalized medicine). Cellular constructs that capture the dynamics of cell-cell interactions in inflammation and drug responses in brain and lung have been developed, 3-5 but correlating data from these constructs with biomarker data from clinical trials will require further research efforts and considerable improvements in computational resources.

A systems biology approach for engineered cell cultures will also benefit infectious disease studies. Modeling the host response to infection generally requires an animal model to capture the complexity of cell-cell interactions involved, including the immune response. To study infectious agents and potential therapeutics in a human system, engineered cell cultures of the relevant tissues created with human cellsprimary cells from healthy or diseased individuals and induced pluripotent cells-will be a powerful tool in the fight against old foes such as hepatitis as well as newly emerging threats such as Zika.

\section{References}

1. Csermely P, Korcsmaros T, Kiss HJ, London G, Nussinov R. Structure and dynamics of molecular networks: a novel paradigm of drug discovery: a comprehensive review. Pharmacol Ther. 2013 Jun;138(3):333-408.

2. Judson R, Kavlock R, Martin M, Reif D, Houck K, Knudsen T, et al. Perspectives on validation of high-throughput assays supporting 21 st century toxicity testing. ALTEX. 2013;30(1):51-6.

3. Hawkins BT, Grego S, Sellgren KL. Three-dimensional culture conditions differentially affect astrocyte modulation of brain endothelial barrier function in response to transforming growth factor betal. Brain Res. 2015 May 22;1608:167-76.

4. Hawkins BT, Hu T, Dougherty ER, Grego S. Modeling neuroinflammatory effects after chemical exposures in a scalable, three-dimensional cell culture system. Appl In Vitro Toxicol. 2016 Dec 1;2(4):223-34.

5. Sellgren KL, Butala EJ, Gilmour BP, Randell SH, Grego S. A biomimetic multicellular model of the airways using primary human cells. Lab Chip. 2014 Sep 7;14(17): 3349-58.
6. Huh D, Leslie DC, Matthews BD, Fraser JP, Jurek S, Hamilton GA, et al. A human disease model of drug toxicity-induced pulmonary edema in a lung-on-a-chip microdevice. Sci Transl Med. 2012 Nov 7;4(159):159ra147.

7. Fermini B, Hancox JC, Abi-Gerges N, Bridgland-Taylor M, Chaudhary KW, Colatsky T, et al. A new perspective in the field of cardiac safety testing through the comprehensive in vitro proarrhythmia assay paradigm. J Biomol Screen. 2016 Jan;21(1):1-11.

8. Khetani SR, Kanchagar C, Okechukwu U, Krzyzewski S, Moore A, Shi J, et al. Use of micropatterned cocultures to detect compounds that cause drug-induced liver injury in humans. Toxicol Sci. 2013 Mar;132(1):107-17.

9. Wilmer MJ, Ng CP, Lanz HL, Vulto P, Suter-Dick L, Masereeuw R. Kidney-on-a-chip technology for drug-induced nephrotoxicity screening. Trends Biotechnol. 2016 Feb;34(2):156-70.

10. Strauss DG, Bilnova K. Clinical trials in a dish. Trends Pharmacol Sci. 2017 Jan;38(1):4-7.

11. Martin F, Sewer A, Talikka M, Xiang Y, Hoeng J, Peitsch MC. Quantification of biological network perturbations for mechanistic insight and diagnostics using twolayer causal models. BMC Bioinform. 2014 Jul 11;15:238.

12. Stokes CL, Cirit M, Lauffenburger DA. Physiome-on-a-chip: the challenge of "scaling" in design, operation, and translation of microphysiological systems. CPT Pharmacometrics Syst Pharmacol. 2015 Oct;4(10):559-62.

13. Li XL, Oduola WO, Qian L, Dougherty ER. Integrating multiscale modeling with drug effects for cancer treatment. Cancer Inform. 2016 Jan 13;14(Suppl 5):21-31.

14. Morris MK, Clarke DC, Osimiri LC, Lauffenburger DA. Systematic analysis of quantitative logic model ensembles predicts drug combination effects on cell signaling networks. CPT Pharmacometrics Syst Pharmacol. 2016 Oct;5(10):544-53.

15. Silva A, Lee B, Clemens DL, Kee T, Ding X, Ho C, et al. Output-driven feedback system control platform optimizes combinatorial therapy of tuberculosis using a macrophage cell culture model. Proc Natl Acad Sci U S A. 2016 Apr 12;113(15):E2172-9.

16. Grego S, Dougherty ER, Alexander FJ, Auerbach SS, Berridge BR, Bittner ML, et al. Systems biology for organotypic cell cultures. ALTEX. 2017 Apr;34(2):301-10.

17. Mueller AJ, Tew SR, Vasieva O, Clegg PD, Canty-Laird EG. A systems biology approach to defining regulatory mechanisms for cartilage and tendon cell phenotypes. Sci Rep. 2016 Sep 27;6:33956.

\section{About the Authors}

Brian T. Hawkins, PhD, is a research biologist in RTI's Engineered Materials, Devices, and Systems (EMDS) group.

Sonia Grego, PhD, is a senior research scientist in RTI's EMDS group, and directs RTI's research efforts in engineered cell cultures.

\section{Acknowledgments}

The authors would like to thank RTI Distinguished Fellows Brian Stoner and Dorota Temple for their support of this area of research. This brief was completed with support from RTI internal funding.

RTI Press Research Briefs and Policy Briefs are scholarly essays on policy, methods, or other topics relevant to RTI areas of research or technical focus.

RTI International, 3040 East Cornwallis Road, PO Box 12194 Research Triangle Park, NC 27709-2194 USA

\section{+1.919.541.6000_rtipress@rti.org www.rti.org}

C2017 RTI International. All rights reserved. Credit must be provided to the author and source of the publication when the content is quoted. No part of this publication may be reproduced in any form by any electronic or mechanical means without permission in writing from the publisher. RTI International is a registered trademark and a trade name of Research Triangle Institute.

RTI Press publication RB-0015-1706 www.rti.org/rtipress 